\title{
A new tool for the seismic investigation of $\gamma$ Doradus stars
}

\author{
A. Moya ${ }^{2}$, J. C. Suárez ${ }^{1}$, P. J. Amado ${ }^{1,3}$, S. Martín-Ruíz ${ }^{1}$, A. Grigahcène ${ }^{1}$, R. Garrido ${ }^{1}$ \\ 1 Instituto de Astrofsica de Andaluca (CSIC), Granada, Spain \\ 2 LESIA, Observatoire de Paris-Meudon, UMR8109, France \\ ${ }^{3}$ European Southern Observatory, Casilla 19001, Santiago 19, Chile.
}

\begin{abstract}
In this work, a generic overview of the Frequency Ratio Method (FRM) is presented. The method is based on the first-order asymptotic g-mode expression given by Tassoul (1980). It is particularly adapted for obtaining asteroseismological information of $\gamma$ Doradus pulsating stars showing at least three pulsation frequencies. The method provides an identification of the radial order $n$ and degree $\ell$ of observed frequencies and an estimate of the integral of the buoyancy frequency (Brunt-Väisälä) weighted over the stellar radius along the radiative zone. Some insights on its applicability to observed frequencies of rotating $\gamma$ Doradus stars are also mentioned.
\end{abstract}

\section{Introduction}

$\gamma$ Doradus stars have been defined by Kaye et al. (1999) as a class of stars pulsating in high- $n$, low- $\ell, g$ modes with very low photometric amplitude, lying on or just to the right of the cool border at the lower part of the Cepheid instability strip. During the last years, tens of new candidates have been observed and catalogued (Mathias et al. 2004, Martín et al. 2003 and references therein).

Only recently, Moya et al. (2005), hereafter Paper I, and Suárez et al. (2005), hereafter Paper II, developed a method for providing useful information for asymptotic $g$-mode pulsators like the $\gamma$ Dor stars. The method is based on the work by Tassoul (1980), who investigated the asymptotic representation of high-frequency $p$-modes and low-frequency $g$-modes associated with low-degree spherical harmonics. The first attempt at obtaining information of a real star by using these $g$-mode asymptotic developments was carried out by Provost \& Berthomieu (1986) adapting the second order asymptotic theory of Tassoul (1980) to the singular case of the Sun. They argued that the study of the $g$ modes of a star would provide information about the physical conditions of the stellar core. At the same time, Kawaler (1987) and Kawaler \& Bradley (1994) started to investigate the internal structure of the PG 1159 hot white dwarf stars through the same asymptotic $g$-mode theory. By using the fully radiative star expression, they obtained stellar properties comparing mode differences of consecutive orders.

For the case of $\gamma$ Dor stars, the FRM supplies additional constraints to the set of classical observables by using information inferred from ratios of the pulsation frequencies measured. Therefore, the set of parameters $\mathcal{P}$ provided by classical observables is increased to

$$
\mathcal{P}=\mathcal{P}_{\Omega}\left(Z, g, L, T_{\text {eff }} ; \frac{f_{o, i}}{f_{o, j}}, \mathcal{I}, \Omega\right)
$$


where $Z$ represent the relative metal abundance, $g$ the gravity, $L$ the luminosity and $T_{\text {eff }}$ the effective temperature. The new constraints are given by $f_{o, i}$ and $f_{o, j}$, which represent the observed frequencies, $\mathcal{I}$, the integral of the Brunt-Väisälä frequency and $\Omega$, the rotational velocity of the star.

In the present work, a general review of the FRM, together with some results on its applicability to rotating $\gamma$ Doradus stars and an application to real stars is provided.

\section{The FRM}

$\gamma$ Doradus stars are found to pulsate in the low frequency g-mode asymptotic regime. There are several analytical forms for obtaining the numerical value of the pulsational period as a function of the radial order $n$, the spherical order $\ell$, different constants and equilibrium quantities, all of these forms being slightly different and being applied for different internal stellar structures. The FRM is based on the following analytical form proposed by Tassoul (1980)

$$
\sigma_{n, \ell}^{a} \approx \frac{[\ell(\ell+1)]^{1 / 2}}{(n+1 / 2) \pi} \mathcal{I}
$$

for a non-rotating star similar in internal structure to that of $\gamma$ Doradus stars. For certain conditions, the use of this expression will not have a significant incidence when applied to a slow-to-moderately rotating $\gamma$ Doradus star (see, Paper II). An exaustive study of the errors commited when using asymptotic theory compared with theoretical predictions with and without rotation, the study of different possible confusing scenarios and the applicability limits of the FRM can be found in Paper I and Paper II and some results will be summarized below. In the rest of this section, only low values for the rotation rate will be assumed in order to remain close to the non-rotation hypothesis.

Let us consider two modes $\sigma_{\alpha_{1}}$ and $\sigma_{\alpha_{2}}$, with $\alpha_{1} \equiv\left(n_{1}, \ell_{1}\right)$ and $\alpha_{2} \equiv\left(n_{2}, \ell_{2}\right)$ respectively. For the sake of simplicity, the same mode degree $\ell_{1}=\ell_{2}=\ell$ of a non-rotating pulsating star showing adiabatic $g$ modes in the asymptotic regime is considered. Their eigenfrequencies can be approximated by Eq. 2 which is model dependent through the integral $\mathcal{I}$. Regarding $\mathcal{I}$ as constant $\left(\mathcal{I}_{\alpha_{2}}=\mathcal{I}_{\alpha_{1}}=\mathcal{I}\right)$, the ratio of $\sigma_{\alpha_{1}}$ and $\sigma_{\alpha_{2}}$ can be approximated by

$$
\frac{\sigma_{\alpha_{1}}}{\sigma_{\alpha_{2}}} \approx \frac{n_{2}+1 / 2}{n_{1}+1 / 2}
$$

An estimate of the radial order ratios of these frequencies can thus be obtained. A value of the integral can also be deduced from observations $\mathcal{I}_{\text {obs }}$, provided that a guess value of the mode degree $\ell$ is assumed. Constraints on models will come from the consistency of: 1 ) the radial order identification corresponding to the observed ratios; 2) their corresponding order $\ell$ and 3 ) the observed Brunt-Väisälä integral.

On the other hand, the assumption of equal $\ell$ for all the observed modes is not imperative for this procedure. Additional information of $\ell$ 's provided by spectroscopy or multicolor photometry can be exploited through the following expression (see Tassoul 1980)

$$
\frac{\sigma_{n_{1}, \ell_{1}}}{\sigma_{n_{2}, \ell_{2}}} \approx \frac{n_{2}+1 / 2}{n_{1}+1 / 2} \frac{\sqrt{\ell_{1}\left(\ell_{1}+1\right)}}{\sqrt{\ell_{2}\left(\ell_{2}+1\right)}}
$$

which is the extended form of Eq. 3.

The efficiency and utility of this method depends on the number of observed frequencies $\mathrm{N}_{f}$. The larger $\mathrm{N}_{f}$ is the lower the number of naturals verifying Eq. 3. It can be shown that the method becomes useful for $\mathrm{N}_{f} \geq 3$. 


\section{Effect of rotation on the FRM}

As shown by observations, $\gamma$ Dor stars rotate in average relatively rapid. Unavoidably, the role that rotation can play for the applicability of the FRM may be significant and this was therefore exhaustively examined in Paper II. There, the validity of the perturbative approach for computing the adiabatic oscillating frequencies of $g$ modes in the asymptotic regime was checked. The effect of rotation on the observational Brunt-Väisälä frequency integral was also studied. Finally, and even more important from the practical point of view, the problem of disentangling rotational multiplet-like structures from the large separation expected for high-order gravity modes in the asymptotic regime was identified and studied. A maximum value for $\Omega$ of the order of or less than $70 \mathrm{~km} \mathrm{~s}^{-1}$ was found as the limit for the rotational velocity which could allow extraction of reliable results from the method. In such cases, the error of the FRM increases one order of magnitude respect to the typical errors already taken into account in the hypothesis of non-rotation.

Concerning the multiplet-like structures, all the possible confusing scenarios were investigated and it was found that, provided any additional information on the mode degree $\ell$, the FRM would be discriminant for $m=0$ modes. For such cases, any misinterpretation induced by the presence of rotationally split multiplet-like structures is avoided. When $\ell$ is a priori unknown, such discrimination is not ensured. Nevertheless, it was shown that the possibility of a confusing scenario was rather unlikely.

\section{The first results}

The first results have been obtained for the $\gamma$ Doradus stars HD 12901 and HD 48501 recently observed by Aerts et al. (2004). In that paper, the authors report three oscillation frequencies for each star, fulfilling thus the main condition for applying the FRM. In the case of HD 48501 with a moderate rotational velocity $\left(v \sin i \sim 30 \mathrm{~km} \mathrm{~s}^{-1}\right)$ this star is particularly suitable to illustrate how the FRM works in the presence of moderate rotation.

\subsection{HD 12901}

For this star, nine possible mode identifications $(n, \ell)$ as well as the corresponding BruntVäisälä integral estimates were obtained using the FRM. From standard constraints on models coming from fundamental parameters (mainly $\log g, T_{\text {eff }}$ and metallicity), nine sets of representative models were selected. In Fig. 1 the selected best models are depicted in a theoretical $\log g-T_{\text {eff }}$ diagram. As can be seen, the surface containing the possible representative models (shaded area) of HD 48501 is significantly smaller than the observational error box.

From these models, theoretical frequencies computed for the nine mode identifications obtained were compared with observations. This lowered the total number of representative models of HD 12901 to six. Furthermore, recent multicolor photometry results for this star given by Aerts et al. (2004) suggests the spherical order to be $\ell=1$. This drastically reduces the number of valid models fitting the observations to only three.

\subsection{HD 48501}

In order to check the FRM in presence of slow-to-moderate rotation, we have applied it to the three observed frequencies of the slowly rotating $\left(v \sin i=29 \mathrm{~km} \mathrm{~s}^{-1}\right) \gamma$ Doradus star HD 48501.

The models fulfilling the corresponding $\left(n_{i}, \mathcal{I}\right)$ predictions were found to be inconsistent with the observational constraints for $\ell=1$ modes, in particular with the observed metallicity. However, for $\ell=2$ modes, the FRM predicts models with masses from 1.4 to $1.6 \mathrm{M}_{\odot}$ and radial orders in the range $n=[38,47]$. Furthermore, when analysing the observed frequencies 


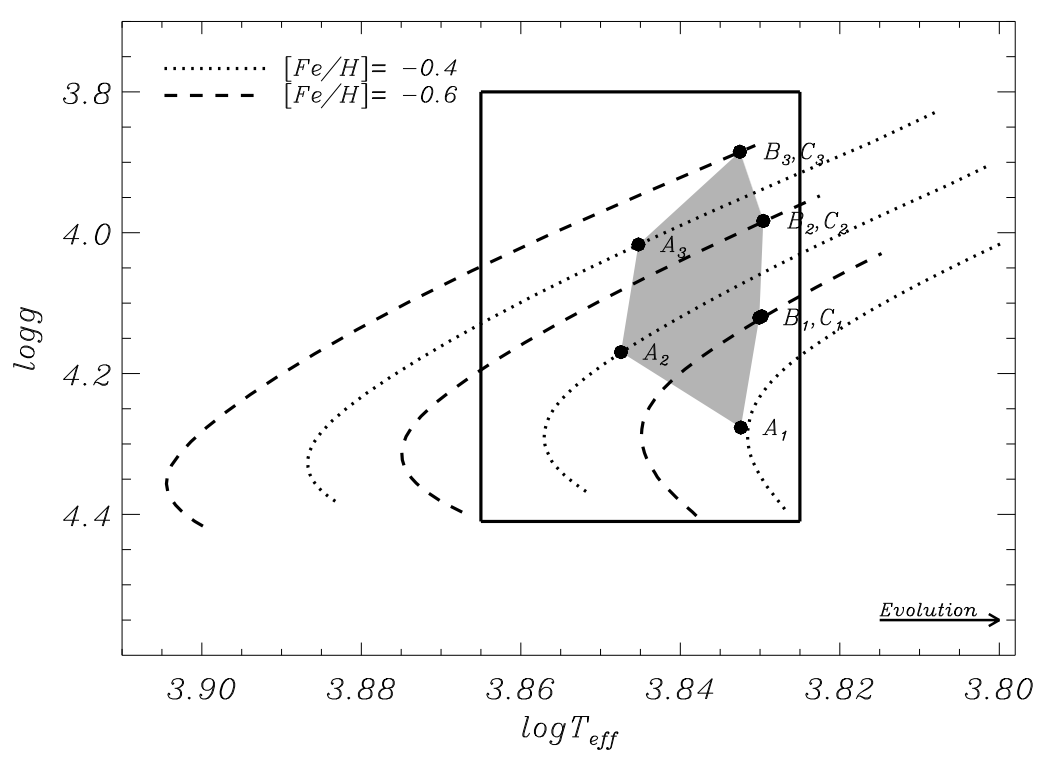

Figure 1: Surface gravity as a function of the effective temperature of selected best models representative of HD 12901. Evolutionary tracks of concerned models are also displayed: dotted lines represents tracks for models with $[\mathrm{Fe} / \mathrm{H}]=-0.4$ and dashed lines those for models with $[\mathrm{Fe} / \mathrm{H}]=-0.6$. The shaded area represents the predicted possible location of representative models of HD 12901 as compared with the observational error box.

as belonging to a rotational induced $\ell=1$ triplet, solutions reproducing the observed average splitting and the triplet average asymmetry have been found to be plausible for a $1.70 \mathrm{M}_{\odot}$ model.

\section{Conclusions}

This method constitutes an important step toward the modal identification for $\gamma$ Doradus stars. It is based on the first order asymptotic relation for the low-frequency g-mode eigenvalues of stellar pulsations, yielding the radial and spherical orders as well as the Brunt-Väisälä integral. The method is developed under the assumptions of adiabaticity and no rotation, and is based on information gathered from ratios of observed frequencies. It has been shown that the FRM can be applied to stars showing at least 3 frequencies.

The study of the role of the rotation in the FRM (Paper II) reveals that reliable results can be obtained for objects with rotational velocities up to $70 \mathrm{~km} \mathrm{~s}^{-1}$. Given the spherical degree $\ell$ identification, the FRM may be discriminant for $m=0$ modes, and any misinterpretation with rotationally induced split multiplet-like structures may be avoided.

Improvements coming from the pulsational behaviour of $\gamma$ Doradus stars are likely to complete the method. Particularly, constraints on unstable modes considering a convectionpulsation interaction can be obtained (work in preparation) from recent theoretical develop- 
ments of Grigahcène et al. (2004) and Dupret et al. (2004).

Acknowledgments. JCS acknowledges financial support by the PNAYA (National Plan of Astronomy and Astrophysics, Spain), within the project AYA2003-04651. Also by the Spanish "Consejería de Innovación, Ciencia y Empresa" from the "Junta de Andalucía" local government, and by the Spanish Plan Nacional del Espacio under project ESP2004-03855C03-01.

\section{References}

Aerts, C., Cuypers, J., De Cat, P., et al. 2004, A\&A, 415, 1079

Dupret, M. A., Grigahcène, A., Garrido, R. et al., 2004, A\&A, 414, 17

Grigahcène, A., Dupret, M.-A., Garrido, R., Gabriel, M., \& Scuflaire, R. 2004, Comm. in Asteroseismology, Vol. 145, 9

Kawaler, S. D. 1987, IAU Colloq. 95: Second Conference on Faint Blue Stars, 297

Kawaler, S. D. \& Bradley, P. A. 1994, ApJ, 427, 415

Kaye, A. B., Handler, G., Krisciunas, K., Poretti, E., \& Zerbi, F. M. 1999, PASP, 111, 840

Martín, S., Bossi, M., \& Zerbi, F. M. 2003, A\&A, 401, 1077

Mathias, P., Le Contel, J.-M., Chapellier, E., et al. 2004, A\&A, 417, 189

Moya A., Suárez J. C., Amado P. J., Martin-Ruíz S., Garrido R., 2005, A\&A, 432, 189 (Paper I)

Provost, J. \& Berthomieu, G. 1986, A\&A, 165, 218

Suárez J. C., Moya A., Martin-Ruíz S., Amado P. J., Grigahcène, A., Garrido R., 2005, A\&A Submitted (Paper II)

Tassoul, M. 1980, ApJS, 43, 469 
